\title{
Erupted abscess cavity and stenosed pulmonary homograft in repeated radical sternotomies: Anaesthesia and haemodynamics control
}

\author{
Yi Lin. Lee ${ }^{1, *}$, Shah Shitalkumar Sharad ${ }^{2}$, Keng Tiong Jerry Tan ${ }^{3}$, Kothandan Harikrishnan ${ }^{4}$ \\ ${ }^{1}$ Senior Resident, ${ }^{2}$ Consultant, ${ }^{3}$ Associate Consultant, ${ }^{4}$ Senior Consultant, Dept. of Anaesthesia, Singapore Ganeral Hospital, \\ Singapore
}

*Corresponding Author:

Email: Yilin.lee@mohh.com.sg

Received: $03^{\text {rd }}$ July, 2017

Accepted: 09 ${ }^{\text {th }}$ August, 2017

\begin{abstract}
Congenital heart disease affects approximately $1 \%$ of the population and is the leading cause of birth defect associated infant illness and death. The most common cyanotic heart disease is tetralogy of Fallot (TOF) which is characterized by overriding of aorta, ventricular septal defect (VSD), pulmonary stenosis and right ventricular hypertrophy. TOF are repaired early in infancy and involves closure of the VSD and repair or replacement of the pulmonary valve to improve right ventricular outflow. A potential and significant complication is stenosis of the pulmonary homograft. We discuss a young patient with previous sternotomies for repair of TOF presenting for his $4^{\text {th }}$ redo-sternotomy for an infective abscess near his old pulmonary homograft. This case presents to us multiple challenges such as a septic patient, previous sternotomies and a potential for intraoperatively massive blood loss.
\end{abstract}

Keywords: Anaesthesia, Cardiac, Heart, Redo-sternotomy, Sepsis.

\section{Introduction}

With the improvement of medical technology and surgical techniques, paediatric patients with traditionally fatal cyanotic congenital cardiac diseases are surviving into young adulthood. In the United States alone, about 20,000 open heart surgeries are performed with $>85 \%$ of infants expected to reach adulthood. ${ }^{1}$ These patients will require lifelong medical surveillance. Unfortunately, their growing-up years is often complicated by infections, valve failures or arrhythmias. ${ }^{2}$ The most common universal approach to reconstruct the RVOT is by using the pulmonary homograft. However, significant transvalvular gradients may develop and this was believed to be either an aftereffect of an immune-mediated response to the homograft $^{3}$ or increased cellular viability, which predisposes to late homograft stenosis. ${ }^{4}$ Patients with more than two previous sternotomies were also at high risk of accidental cardiovascular trauma during dissection for further surgeries. ${ }^{5}$ We discuss the anaesthetic considerations for such patient presenting to us for redo-cardiac surgeries.

\section{Case Description}

We report a 21-year-young patient with previously repaired tetralogy of Fallot, presenting for $4^{\text {th }}$ redosternotomy for infected pulmonary homograft. Following total repair at the age of 3, had undergone revision of the proximal anastomosis of pulmonary homograft with anterior pericardial patch in 2012 for severe pulmonary regurgitation and a pulmonary graft revision in 2015 for right ventricular outflow tract (RVOT) obstruction at the anastomotic site.
He presented with prolonged fever of unknown origin and was found to have an echogenic lesion in the distal RVOT extending into the pulmonary homograft with significant homograft stenosis on transthoracic echocardiography (TTE) [Fig. 1]. The mean pressure gradient across the graft was $47 \mathrm{mmHg}$. The patient was initially treated conservatively with broad-spectrum antibiotics for presumptive infected pulmonary homograft. Despite 8 weeks of inpatient parenteral antibiotics, source control was deemed inadequate. Surgery was advised and the patient was agreeable for redo-sternotomy, drainage of pus and abscess cavity and open inspection of pulmonary homograft.

Prior to induction of anaesthesia, invasive pressure lines (radial intra-arterial line, 4 lumen left internal jugular vein central line) and standard monitoring with a 5 lead electrocardiogram (ECG), pulse oximetry and bispectral index (BIS) was established. A large bore $16 \mathrm{G}$ intravenous cannula was inserted at his right external jugular vein at surgeon's request to be used for possible access to the superior vena cava in the event of torrential bleeding during sternotomy. Balanced anaesthesia was induced carefully with titrated doses of midazolam, etomidate, fentanyl and rocuronium and maintained with sevoflurane in oxygen and air. Hemodynamics were maintained at a mean arterial pressure of $>65 \mathrm{mmHg}$, BIS $40-60, \mathrm{SpO} 2>98 \%$ and end-tidal $\mathrm{CO} 230-35 \mathrm{mmHg}$, with intermittent use of adrenaline and noradrenaline infusions. Maintenance drip was via a Hartmann's solution. Femoral vessels were cannulated for cardiopulmonary bypass (CPB). Upon entering the pericardium, turbid fluid was noticed and the other finding was of a defect and haematoma 
within the wall of the homograft with a sinus tracking to the abscess cavity posterior to the main pulmonary artery. The abscess cavity was debrided and temporary chest closure was performed with intention of a relook after 2 days. Patient was transferred to the intensive care unit (ICU) intubated, ventilated and sedated. Estimated blood loss was $400 \mathrm{ml}$ and no blood conservation strategies were utilized.

Two days later, repeat exploration was performed for debridement and removal of homograft and insertion of new pulmonary homograft. Similarly, femoral cannulation was performed for CPB. The abscess cavity appeared clean and the in situ pulmonary homograft was removed due to concerns of graft infection. Following implantation of the new homograft, transoesophageal echocardiography (TOE) showed high gradients between the mean pulmonary artery and the bifurcation of the main pulmonary artery. The new homograft was reopened for inspection and no obvious stenosis nor kinks were observed. Chest was closed and patient sent back to ICU. Estimated blood loss was $500 \mathrm{ml}$ and again no blood conservation strategies were needed.

Patient recovered well in the ICU and was extubated 2 days later. TTE showed a persistent narrowing distal to the graft but suggested that this could be due to in- folding of the curved segment of the homograft as it was obtained from an aortic source. He required a total of 6 weeks of intravenous antibiotics and was discharged 2 months into his stay.

\section{Discussion}

Patients who undergo corrective surgery for TOF may require repeat surgeries in the long-term to treat right ventricular outflow tract obstructions (RVOTO), VSD leaks, infective endocarditis, pulmonary regurgitation, and other complications. Due to medical advances, these patients are surviving into adulthood and their numbers have been steadily increasing. ${ }^{6-10}$

The main worry during reoperation is the inherent risk of the procedure itself. The factual reported mortality rates for reoperation have been relatively high at $7.1-15.5 \%,{ }^{7-9}$ with the most frequent perioperative morbidity accidental cardiovascular trauma during dissection. In a study by Morales, ${ }^{11}$ he found that significant haemorrhages occurred in $1.5 \%$. This occurs because of adhesions between the heart and mediastinum which may be injured during dissection. In his study, $76 \%$ of injuries occurred to the cardiac structures, grafts and conduits. Patients are particularly susceptible to injuries during sternal entry and prepump dissection. Surgical strategies including specialised instruments such as the oscillating saws, as well as polytetrafluoroethylene surgical membrane and tissue sealants, have helped to prevent adhesion formation and hence minimize bleeding.
In anticipation of massive haemorrhage, a twoprong approach should be considered. Firstly, steps for a timely and adequate resuscitation; and secondly, blood conservation methods. To address the former, patients should have a large bore intravenous access capable of rapid infusion. A fluid management system must be available for rapid transfusion of large volumes of warmed fluid and blood products readily available within the operating theatre complex. A robust massive transfusion protocol should be in place depending on individual institution's guideline. Blood conservation techniques include aggressive correction of coagulopathy, meticulous surgical techniques and the use of a cell saver.

Blood transfusion has been associated with increased morbidity and mortality with a study by Engoren ${ }^{12}$ showing that patients who were transfused blood products had twice the 5-year mortality compared to those who were not. Hence it is impertinent to avoid unnecessary haemodilution by employing preventive strategies such as minimising fluid therapy, reducing priming volume of the bypass circuit and the use of cell saver in repeat surgery. ${ }^{13}$

The use of a cell saver is controversial in cases of malignancy and sepsis as it is thought that the contaminated cell salvaged blood products will serve as an infective/malignant source. However, there is no available data supporting this claim in the literature even though bacteria contamination of cell salvaged blood appears common. ${ }^{14-15}$ A double suction set-up (one suction line is connected to the cell salvage reservoir and is used for suctioning blood; while the other is connected to the regular wall suction and is used for aspirating the contaminant) may be used to reduce contamination. ${ }^{16}$ The risks of contaminated cell salvaged blood products are also reduced due to both the use of prophylactic antibiotics and the removal of contaminants by the machine.

Unfortunately, graft infection is a known complication of (bio) prosthetic valves. Homografts are obtained from cadavers and are more resistant to developing endocarditis compared to other valves. In addition, homografts negate the need for anticoagulation and do not cause haemolysis. Sepsis leads to vasodilatation, a hyperdynamic circulation with lower cardiac reserves, and possible multi-organ insults such as acute respiratory distress syndrome (ARDS) with high ventilatory requirements, acute renal impairment and coagulopathy. A thorough preoperative assessment specifically targeting these areas should be performed with risk stratification and counselling done. Patients may also be on multiple broad spectrum antibiotics which should be continued during the perioperatively phase. Timely administration of antibiotics during the peri-operative period is crucial and early involvement of an infectious disease specialist should be considered. 
The need for meticulous surgical techniques coupled with a distorted cardiac anatomy will result in a prolonged operation time and duration on bypass. Prolonged cardiac bypass duration leads to multisystemic disorders. It is associated with an increased risk of postoperative mortality, pulmonary, renal, and neurologic complications, multiorgan failure, reoperation for bleeding, and multiple blood transfusions. ${ }^{17}$ Therefore, it is necessary to discuss the extent of procedure with the surgeons, and maintain good communication with them throughout the perioperative period. Unexpected prolonged surgeries should be staged after discussion with the surgeons and returned another day.

While TEE remains the gold standard for diagnosis of infective endocarditis, up to half may still be missed. ${ }^{18}$ Nonetheless, it remains useful during cardiac surgery as it can help to delineate the valve and conduit function. TEE in a patient with repaired congenital cardiac disease is difficult as their anatomies are often distorted. A specialised cardiologist with interest in adult congenital heart disease should hence be consulted early for assistance.

\section{Conclusion}

The number of young patients presenting for repeated cardiac re- surgery is invariably raising and we need to be prepared for them. These patients have fundamentally altered cardiac physiology and anatomy and will pose challenges to both the anaesthetists and surgeons. A multi-disciplinary approach coupled with meticulous blood conservation strategies will positively influence the outcome of these patients. IRB and patient approval were obtained.

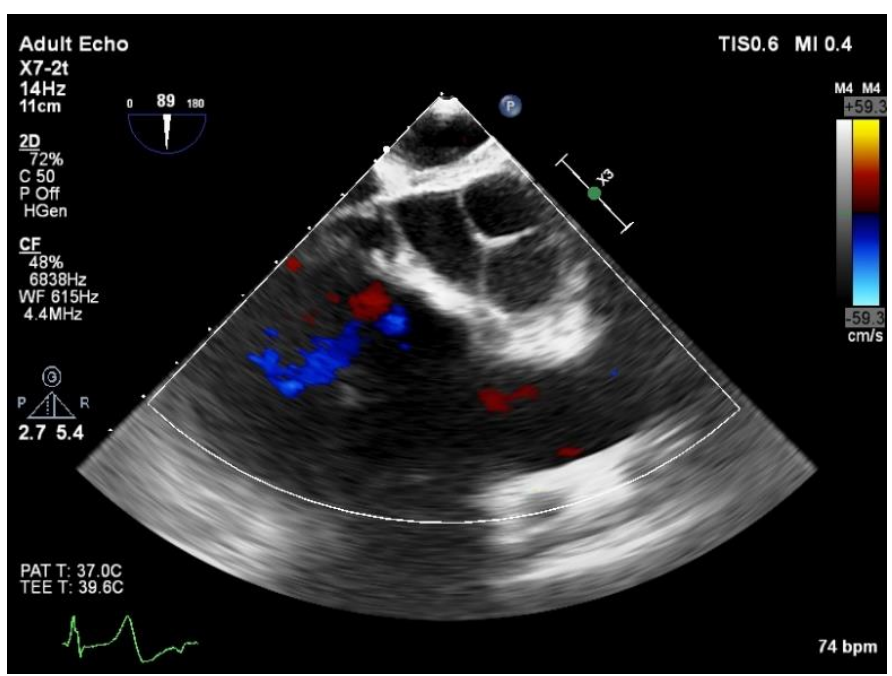

Fig. 1: TOE view of the RVOT showing a hyperechoic lesion in the distal RVOT

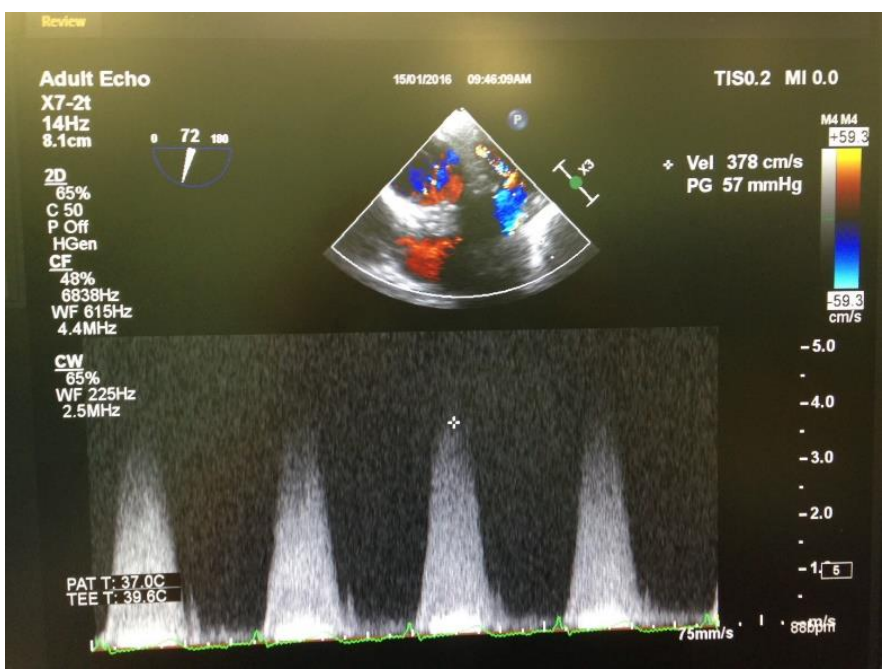

Fig. 2: TOE view of the RVOT showing high gradients between the mean pulmonary artery and the bifurcation of the main pulmonary artery 


\section{References}

1. Moodie DS. Adult congenital heart disease. Curr Opin Cardiol. 1994;9:137-42.

2. JK Perloff, CA Warnes. Challenges posted by adults with repaired congenital heart disease. Circulation 2001;103:2637-43.

3. Moidl R, Simon P, Kupilik N, et al. Increased flow velocities in oversized homografts in patients after the Ross procedure. Eur J Cardiothorac Surg 1997;12:56972.

4. Ehud Raanani, MD, Terrence M. Yau, Ann Thorac Surg 2000;70:1953-7.

5. Takaaki Sugita, MD, Yuichi Ueda, MD, Masahiko Matsumoto, MDAnn Thorac Surg 2000;70:1507-10.

6. Castañeda AR, Sade RM, Lamberti J, Nicoloff DM. Reoperation for residual defects after repair of tetralogy of Fallot. Surgery 1974;76:1010-7.

7. Uretzky G, Puga FJ, Danielson GK, Hagler DJ, McGoon DC. Reoperation after correction of tetralogy of Fallot. Circulation 1982; 66 (Suppl 1): I-202-8.

8. Isomura $\mathrm{T}$, Hisatomi $\mathrm{K}$, Andoh $\mathrm{F}$, et al. Reoperation following total repair of congenital heart disease. Jpn Circ J 1991;55:453-8.

9. Pome G, Rossi C, Colucci V, et al. Late reoperations after repair of tetralogy of Fallot. Eur J Cardiothorac Surg 1992;6:31-5.

10. Oechslin EN, Harrison DA, Harris L, et al. Reoperation in adults with repair of tetralogy of Fallot: indications and outcomes. J Thorac Cardiovasc Surg 1999;118:245-51.

11. D Morales, E Williams, R John. Is resternotomy in cardiac surgery still a problem? Interactive CardioVascular and Thoracic Surgery 11(2010):277-86

12. MC Engoren, RH Habib, A Zacharias, TA Schwann, CJ Riordan, SJ Durham. Effect of blood transfusion on longterm survival after cardiac operation. The Annals of thoracic Surgery Oct 2002 74(4):1180-6

13. NC Hwang. Preventive Strategies for Minimizing Hemodilution in the Cardiac Surgery Patient During Cardiopulmonary Bypass. J Cardiothorac Vasc Anesth. 2015 Dec;29(6):1663-71.

14. Bland LA, Villarino ME, Arduino MJ, et al. Bacteriologic and endotoxin analysis of salvaged blood used in autologous transfusions during cardiac operations. J Thorac Cardiovasc Surg. 1992;103:582-8.

15. Kang Y, Aggarwal S, Pasculle AW, et al. Bacteriologic study of autotransfusion during liver transplantation. Transpl Proceed. 1989;21:35-8.

16. SA Esper, JH Waters. Intra-operative cell salvage: a fresh look at the indications and contraindications. Blood Transfus 2011;9:139-47.

17. S Salis, VV Mazzanti, G Merli, L Salvi, CC Tedesco, et al. Cardiopulmonary bypass duration is an independent predictor of morbidity and mortality after cardiac surgery. Jounral of Cardiothoracic and Vascular Anaesthesia Dec 2008;22(6):814-22.

18. EE Hill, P Herijgers, $P$ Claus, $S$ Vanderschueren, WE Peetermans, et al. Abscess in infective endocarditis: the value of transesophageal echocardiograpy and outcome: a 5 year study. American Heart Journal Nov 2007;154(5):923-8. 